\title{
Pain Prevalence, Management and Interference Among University Students in South Korea: An Exploratory Cross-Sectional Study
}

\author{
Hee Jun Kim (D) \\ Sunjoo Boo (D) \\ Timothy J Meeker ${ }^{2}$ \\ 'Research Institute of Nursing Science, \\ College of Nursing, Ajou University, \\ Suwon, 16499, Gyeonggi, Republic of \\ Korea; ${ }^{2}$ Department of Neurosurgery, \\ Johns Hopkins Medical Institute, \\ Baltimore, MD, 2I287, USA
}

Background: Pain is a global health issue with a significant impact on young adults. Adverse effects caused by inappropriate pain management among university students are related to poor mental/physical health. This study aimed to explore pain prevalence, management, and interference among university students in South Korea.

Methods: Pain intensity, painful body areas, pain management, and pain interference were measured in a convenience sample of 404 students. Descriptive statistics are reported, and a multivariable binomial logistic regression was conducted to reveal factors associated with pain interference.

Results: The prevalence of acute and chronic pain was $73.5 \%$, while $7.8 \%$ reported chronic pain ( $\geq 3$ months). Half of university students who experienced pain reported at least four painful body areas. The average pain intensity during the past 6 months was $4.8 / 10$. About $56 \%$ of university students who experienced pain used over-the-counter pain pills for pain management. Rest and massage were the most used non-pharmacological pain management strategies. Mood was the most reported pain interference complaint amongst university students. Greater pain interference was associated with longer pain duration, more painful body areas, and greater pain intensity.

Discussion: Pain is highly prevalent among South Korean university students. Pain management programs, including education about appropriate methods of pain relief, should be developed for university students. Attention should be given to university students with widespread acute and chronic pain of high intensity to mitigate the negative impacts caused by pain interference.

Keywords: pain, pain management, prevalence, young adults

\section{Introduction}

Individual classification of actually or potentially injurious events as painful is learned from injury-related experiences during early life that generate activity in peripheral nociceptive afferents. ${ }^{1}$ These pain-related experiences differ throughout the lifetime of individuals as environmental, social, cultural, and biological factors that influence pain, and its subjective experience can change over time. Pain is subjective and variation in pain sensitivity is well documented at the individual level and at the level of race/ethnicity; for example, Asians tend to have higher pain sensitivity compared to non-Hispanic Whites. ${ }^{2}$

The negative impact of pain, including low quality of life, disability, and psychological distress, induces intangible costs both to the individual and to the
Correspondence: Sunjoo Boo Research Institute of Nursing Science, College of Nursing, Ajou University, 164, World Cup-ro, Yeongtong-gu, Suwon, Gyeonggi, 16499, Republic of Korea Tel +82-3I-219-7032

Fax +82312197020

Email sjboo@ajou.ac.kr 
society at large. ${ }^{3}$ These negative impacts are likely important in young adults because they face various stressors, life changes and challenges as they develop their adult lifestyle. In fact, previous studies reported that pain in young adults was associated with disability, lower quality of life, and reduced general work productivity. ${ }^{4}$ Further, pain complaints, especially among university students, were associated with opioid misuse and poorer mental health, including depression. ${ }^{5-7}$

University students may have not yet developed effective coping strategies to manage their pain, which ultimately can exacerbate the negative impact of pain. Appropriate pain management in university students is important to reduce pain-associated long-term adverse effects. Individual beliefs developed from previous experiences, culturally learned traditions, and familial or peer group rituals likely influence university students' pain management. For example, a study found that university students preferred to self-manage their pain using over-the -counter (OTC) medications or self-determined nonpharmacological therapies, as they believe pain is a condition they can self-manage. ${ }^{8,9}$ However, pain selfmanagement in young adults is associated with missed or delayed diagnosis, drug interactions, polypharmacy and medication overuse. ${ }^{10,11}$

Understanding the aspects of life involved in pain interference and the level of pain interference experienced by university students is important to better manage pain and improve health outcomes. Life roles and activities of daily living affected by pain include general activity, mood, walking ability, normal work (eg, work outside the home or housework), relations with other people, sleep, and enjoyment of life. ${ }^{12}$ In addition to these realms, university students with acute or chronic pain may experience interference with academic performance.

The reported prevalence of self-reported acute pain in previous studies has ranged from $40 \%$ to over $90 \%$ among university students. Past studies of pain in university students have focused on specific conditions such as dysmenorrhea, ${ }^{8,13-17}$ orofacial pain ${ }^{18}$ and musculoskeletal pain. ${ }^{6,19-24}$ Other studies focused on a specific group of students such as health students ${ }^{25}$ or music students. ${ }^{26}$ In South Korea, previous studies of pain experience in university students have been limited to examining associations between pain experience and physical activity, ${ }^{27}$ mobile phone use, ${ }^{28,29}$ or internet use. ${ }^{30}$

University students appear vulnerable to a high prevalence of acute pain and have significant negative consequences from pain experiences. Exploring pain prevalence, pain management methods, and pain interference among university students is important to develop appropriate programs to address the negative impact of pain. However, few studies have examined these factors in university students. The purpose of this study was to assess the prevalence of acute and chronic pain, pain management methods and their perceived effectiveness, and pain interference among university students in South Korea. We also aimed to explore factors associated with pain interference in this population.

\section{Materials and Methods Study Design and Sample}

We conducted an exploratory cross-sectional study using anonymous paper-based surveys. A convenience sample of university students was recruited from four universities in South Korea in March 2016. Subjects were eligible to participate in the study if they were students enrolled in the universities during the study period and agreed to participate in the study.

\section{Data Collection Approach}

Trained research assistants visited each university and approached potential participants personally while they were in campus communal spaces such as libraries. The potential participants were informed of the purpose of the research and the voluntary and anonymous nature of the study. Ethical approval was obtained from the institutional review board of Ajou University Medical Center (AJIRBSBR-SUR-15-469). All participants provided informed consent, and this study was conducted in accordance with the Declaration of Helsinki.

\section{Measures}

The survey includes self-report measures of pain (pain prevalence, painful body areas, pain intensity and duration), pain management methods (pharmacological and non-pharmacological), pain interference, and demographic characteristics.

\section{Prevalence of Pain}

To measure the prevalence of pain, participants were asked "Have you had any pain during the last 6 months?". If they answered yes to the question, then they were asked to report pain duration, painful body areas, intensity, and interference. For pain duration, a single item was used: "How long did the pain last?"; and the response categories 
were as follows: less than one day, 1-7 days, 8-30 days, 1-3 months, or 3-6 months. Painful body areas were assessed using a graphic map showing 16 body areas (head, neck, shoulder, chest, stomach, abdomen, dysmenorrhea-related, arms, legs, wrist, ankle, knee, lower back, upper back, elbow, malleolus, and others). Participants were asked to indicate if they had pain in any of these areas, and if they had, they were asked to indicate their pain intensity. Pain intensity was measured using the numeric pain rating scale $(0-10)$, where 0 corresponds to no pain, and 10 corresponds to pain as bad as you can imagine. Cronbach's alpha for pain intensity rating was 0.865 for the current study.

\section{Pain Management}

Pain management methods included in this study were divided into three parts: seeking help from a medical professional, pharmacological, and non-pharmacological. Participants were asked if they had consulted with a general practitioner, physiotherapist, chiropractor, bone setter, or traditional Chinese physician (yes/no). Five items were included in the pharmacological management section asking the participants to report if they had used any OTC medication, topical agents, pain relief patch, or pain relief pills to relieve pain (yes/no). Non-pharmacological management self-report items included were acupressure, acupuncture, cold/heat application, deep breathing, aromatherapy, exercise, massage, music, rest, or traditional Chinese medicine (TCM) including cupping, scraping, or transcutaneous electrical nerve stimulation (TENS). Perceived effectiveness of each pain management method was also measured using a numeric rating scale $(0=$ no relief and $5=$ complete relief).

\section{Pain Interference}

Pain interference indicates the degree to which pain interferes with aspects of a participant's life. ${ }^{12}$ For the current study, a composite index including 8 items was used including items related to the degree of interference with academic performance, general activity, mood, walking ability, normal work, relations with other people, sleep, and enjoyment of life. Each item was assessed using a numeric rating scale $(0=$ no interference and $5=$ interferes completely), averaged and converted to $0-10$ scale for better interpretability. Cronbach's alpha was 0.835 for the current study.

Demographic variables included in the study were age, gender, year in school, area of study, and weekly frequency of physical activity lasting more than 30 minutes. The original survey tool was developed by adopting and modifying from the Brief Pain Inventory-Short Form to make them more suitable for Asian university students. ${ }^{24}$ For this study, the original English version was translated into Korean by two translators and differences reconciled. The reconciled version was translated back into English by an independent, bilingual translator. The new English version was compared with the original English version to check for any loss of meaning. Readability was tested by three university students.

\section{Statistical Analysis}

Summary statistics are reported to provide a description of demographic characteristics, pain prevalence, body areas of pain, and pain intensity for participants who reported pain. Frequencies and percentages are reported for categorical variables, while median, interquartile range, mean, and standard deviation (SD) are reported for continuous variables. Independent $t$-tests were used to test group differences on demographic characteristics between the pain and without pain groups, and chi-square tests were utilized to measure the association between categorical demographic variables and pain prevalence. To assess factors associated with pain interference, a multivariable binomial logistic regression was conducted. The logistic regression analysis only included participants reporting pain in the past 6 months. Data normality was assessed using histograms, normal probability plots and measures of skewness or kurtosis. The composite index of pain interference was dummy coded as $0=\leq 3$ and $1=>3$ for logistic regression by the median-split method due to skewness. Demographic variables, pain duration, painful body area, average pain intensity, use of OTC medication, and use of non-pharmacological method were evaluated as bivariate factors associated with pain interference. Variables that showed trend-level effects $(p<0.1)$ in each bivariate analysis were entered into the multivariable model. A p-value of less than 0.05 was considered statistically significant. All analyses were performed using Stata version 15.1 (StataCorp LLC, College Station, TX, USA).

\section{Results}

\section{Demographic Characteristics}

A total of 404 students participated in the study. Their mean age was 22.4 years $(\mathrm{SD}=2.73)$ and ranged from 17 to 45 years old. Sixty-one percent of the participants 
Table I General Characteristics of Participants $(N=404)$

\begin{tabular}{|c|c|c|c|c|c|}
\hline \multirow[t]{2}{*}{ Characteristics } & Pain $(n=297)$ & No Pain $(n=107)$ & Total $(n=404)$ & \multirow[t]{2}{*}{$\chi^{2}$ or $\mathbf{t}$} & \multirow[t]{2}{*}{$p$} \\
\hline & n (\%) & n (\%) & n (\%) & & \\
\hline Age, mean (SD), range & $22.7(2.90)$ & $21.8(2.04)$ & $22.43(2.73), 17-45$ & 3.04 & 0.003 \\
\hline \multicolumn{6}{|l|}{ Gender } \\
\hline Male & $98(62.40)$ & $59(37.60)$ & I57 (38.96) & & \\
\hline Female & I 98 (80.49) & $48(19.51)$ & $246(61.04)$ & 16.04 & $<0.001$ \\
\hline \multicolumn{6}{|l|}{ Year in school } \\
\hline Freshman & $67(59.82)$ & $45(40.18)$ & II 2 (27.79) & & \\
\hline Sophomore & $8 I(7 I .05)$ & 33 (29.95) & II 4 (28.29) & & \\
\hline Junior & 40 (74.07) & $14(25.93)$ & $54(13.4)$ & & \\
\hline Senior & $89(86.4 I)$ & 14 (I3.59) & $103(25.56)$ & & \\
\hline Graduate school & $19(95.00)$ & I (5.00) & $20(4.96)$ & 24.65 & $<0.001$ \\
\hline \multicolumn{6}{|l|}{ Major } \\
\hline Health related & $91(81.25)$ & $21(18.75)$ & $112(28.28)$ & & \\
\hline Not health related & $200(70.42)$ & $84(29.58)$ & 284 (7I.72) & 4.83 & 0.028 \\
\hline \multicolumn{6}{|l|}{$\begin{array}{l}\text { Frequency of physical } \\
\text { activity lasting more than } \\
30 \text { minutes }\end{array}$} \\
\hline None & I58 (75.24) & $52(24.76)$ & $210(52.63)$ & & \\
\hline I-2 times/week & 97 (76.98) & $29(23.02)$ & $126(31.58)$ & & \\
\hline 3 or more/week & $40(63.49)$ & $23(36.5 \mathrm{I})$ & $63(15.79)$ & 4.36 & 0.113 \\
\hline
\end{tabular}

were female, and $71.7 \%$ of the participants were not in a health-related major (eg, engineering) (Table 1). About half of the participants $(53 \%)$ indicated that they did not exercise more than 30 minutes per week. The pain and nopain groups were significantly different in age, gender, year in school, and area of study. Older age, being female, having more years in school, and being in a health-related area of study were all associated with a higher prevalence of pain $(\mathrm{p}<0.05)$.

\section{Pain Characteristics of the Sample}

Among the university student sample, prevalence of pain was $73.5 \%$ (95\% CI $=[0.689,0.776])$ (Table 2). Among the 297 participants who reported experiencing pain in the past 6 months, the majority (54.2\%) reported experiencing pain for 1-7 days. Twenty-two percent of participants reported experiencing pain less than one day, while $7.8 \%$ reported chronic pain lasting more than 3 months. The most commonly reported painful body area was the shoulders $(55.2 \%)$, followed by head $(45.8 \%)$ and neck $(42.8 \%)$. Forty-seven percent of female participants reported dysmenorrhea. The average pain intensity was $4.8(\mathrm{SD}=1.71)$ on a 10 -point scale, and the median number of painful body areas was four.

\section{Pain Management Methods and Perceived Effectiveness}

About one-third of participants who reported experiencing pain indicated they sought medical help with pain relief (Table 3 ). The most frequently consulted healthcare professional was a general practitioner (20.2\%), followed by physiotherapist (13.1\%), and traditional Chinese physician (10.4\%). Chiropractor had the highest mean score for the perceived effectiveness $(\mathrm{M}=$ 3.33, SD =1.75). For pharmacological management methods, $45 \%$ of participants with pain used OTC medications (eg, Tylenol and ibuprofen). OTC pills were used by $56.2 \%$, while pain relief patches were used by $32 \%$ of participants. More than half of participants (57.2\%) responded they used nonpharmacological methods for pain management. The most frequently used non-pharmacological method was rest $(36.7 \%)$, followed by massage $(29.6 \%)$, acupressure (23.2\%) and exercise (18.2\%). Rest was reported to have the highest mean perceived effectiveness of pain relief, followed by acupuncture $(\mathrm{M}=3.25$, $\mathrm{SD}=1.19 ; \mathrm{M}=3.08, \mathrm{SD}=1.16$ ). 
Table 2 Pain Duration, Areas, and Intensity of University Students Who Experienced Pain in the Past 6 Months $(N=297)$

\begin{tabular}{|c|c|c|c|c|}
\hline Characteristics & $\mathbf{n}$ & $\%$ & \multicolumn{2}{|c|}{$95 \% \mathrm{Cl}$} \\
\hline Prevalence of pain & 297 & 73.5 & \multicolumn{2}{|c|}{$0.689,0.776$} \\
\hline \multicolumn{5}{|l|}{ Duration of pain } \\
\hline Less than one day & 64 & 21.69 & \multicolumn{2}{|c|}{$0.173,0.287$} \\
\hline I-7 days & 160 & 54.24 & \multicolumn{2}{|c|}{$0.485,0.598$} \\
\hline $8-30$ days & 26 & 8.81 & \multicolumn{2}{|c|}{$0.060,0.126$} \\
\hline I-3 months & 22 & 7.46 & \multicolumn{2}{|c|}{$0.049,0.111$} \\
\hline More than 3 months & 23 & 7.79 & \multicolumn{2}{|c|}{$0.052,0.115$} \\
\hline \multirow[t]{2}{*}{ Painful body area } & \multicolumn{2}{|c|}{ Frequency } & \multicolumn{2}{|c|}{$\begin{array}{l}\text { Pain intensity } \\
(0-10 \text { scale })\end{array}$} \\
\hline & $\mathbf{n}$ & $\%$ & $M$ & SD \\
\hline Shoulder & 164 & 55.2 & 4.34 & 1.97 \\
\hline Head & 136 & 45.8 & 4.74 & 1.96 \\
\hline Neck & 127 & 42.8 & 4.42 & 2.07 \\
\hline Dysmenorrhea related & 138 & 46.5 & 5.74 & 2.49 \\
\hline Abdomen & 83 & 27.9 & 5.07 & 2.20 \\
\hline Stomach & 75 & 25.3 & 4.60 & 2.40 \\
\hline Ankle & 70 & 23.6 & 4.33 & 2.38 \\
\hline Wrists & 68 & 22.9 & 3.82 & 1.88 \\
\hline Knee & 68 & 22.9 & 4.57 & 2.22 \\
\hline Lower back & 55 & 18.5 & 4.66 & 1.97 \\
\hline Legs & 39 & 13.1 & 4.83 & 2.35 \\
\hline Chest & 31 & 10.4 & 4.34 & 2.32 \\
\hline Upper back & 31 & 10.4 & 4.34 & 2.10 \\
\hline Arms & 20 & 6.7 & 4.48 & 1.57 \\
\hline Elbow & 15 & 5.1 & 5.07 & 2.27 \\
\hline Malleolus & 13 & 4.4 & 4.67 & 2.46 \\
\hline $\begin{array}{l}\text { Others (eg, jaw, oral, ear, eyes, fingers, } \\
\text { toes) }\end{array}$ & 21 & 7.1 & 4.40 & 2.73 \\
\hline
\end{tabular}

Abbreviations: $\mathrm{Cl}$, confidence interval; IQR, interquartile range.

\section{Pain Interference and Associated Factors}

Mood showed the greatest interference level associated with pain $(\mathrm{M}=2.57, \mathrm{SD}=1.62)$, followed by general activity $(\mathrm{M}=2.25, \mathrm{SD}=1.50)$ (Table 4$)$. Students indicated that pain interfered with normal work, walking ability, and sleep. Average interference scores ranged from 0 to 9, with a median of 3 (IQR: 1.5-4.5). Multivariable binomial logistic regression indicated that more painful body areas $(\mathrm{OR}=2.98, \mathrm{p}=0.003)$, longer pain duration: 1-7 days and more than a week compared to less than
Table 3 Methods of Pain Management and Perceived Effectiveness Among University Students Who Reported Experiencing Pain in the Past 6 Months ( $\mathrm{N}=297)$

\begin{tabular}{|l|c|c|c|c|}
\hline Pain Management Methods & \multicolumn{2}{|l|}{ Frequency } & \multicolumn{2}{c|}{$\begin{array}{l}\text { Perceived } \\
\text { Effectiveness }\end{array}$} \\
\cline { 2 - 5 } & $\mathbf{n}$ & $\%$ & $\begin{array}{c}\text { Mean } \\
\text { SD }\end{array}$ & \\
\cline { 2 - 5 } Seeking medical help & & & $(0-5)$ & \\
General practitioner & 98 & 33.2 & - & - \\
Physiotherapist & 60 & 20.2 & 2.68 & 1.59 \\
Chiropractor & 39 & 13.1 & 2.18 & 1.31 \\
setter & 7 & 2.4 & 3.33 & 1.75 \\
Traditional Chinese physician & 31 & 1.0 & - & - \\
\hline Pharmacological Management & & & & \\
Use of any OTC medication & 134 & 45.1 & - & - \\
Pain relief pills (OTC) & 167 & 56.2 & 3.28 & 1.17 \\
Topical agents & 57 & 19.2 & 2.59 & 1.33 \\
Pain relief patch & 95 & 31.9 & 2.37 & 1.28 \\
Oral intake of Chinese Medicine & 29 & 9.7 & 1.97 & 1.74 \\
\hline Non-pharmacological method & & & & \\
Any non-pharmacological method & 170 & 57.2 & - & - \\
Rest & 109 & 36.7 & 3.25 & 1.19 \\
Massage & 88 & 29.6 & 3.03 & 0.98 \\
Acupressure & 69 & 23.2 & 2.65 & 0.94 \\
Exercise & 54 & 18.2 & 2.76 & 1.13 \\
Heat application & 40 & 13.5 & 2.80 & 1.14 \\
Music & 37 & 12.5 & 2.44 & 0.94 \\
Acupuncture & 36 & 12.1 & 3.08 & 1.16 \\
Deep breathing & 35 & 11.8 & 2.17 & 0.96 \\
TENS & 27 & 9.1 & 2.67 & 1.11 \\
Cold application & 20 & 6.7 & 2.40 & 0.82 \\
Aromatherapy & 5 & 1.7 & 2.20 & 1.10 \\
TCM (eg, Cupping therapy, & 1.3 & 2.00 & 1.41 \\
Scarping) & & & \\
\hline
\end{tabular}

Abbreviations: SD, standard deviation; TENS, transcutaneous electrical nerve stimulation; OTC, over-the-counter drug; TCM, traditional Chinese medicine.

one day $(\mathrm{OR}=3.01, \mathrm{p}=0.013 ; \mathrm{OR}=4.46, \mathrm{p}=0.005)$, and greater pain intensity $(\mathrm{OR}=1.67, \mathrm{p}<0.001)$ were all associated with greater pain interference (Table 5). Female gender, being in a health-related area of study, and using OTC medication that had had significant associations with pain interference in bivariate analyses were not statistically significant in the final multivariable model.

\section{Discussion}

This study explored the prevalence of pain, frequency of specific painful body areas, pain intensity, pain management methods and their perceived effectiveness, and pain interference among university students in South Korea. 
Table 4 Pain Interference Among the University Students Who Experienced Pain in the Past 6 Months ( $=297)$

\begin{tabular}{|l|c|c|}
\hline Characteristics & Mean (0-5) & SD \\
\hline Academic performance & 0.92 & 1.32 \\
General activity & 2.25 & 1.50 \\
Mood & 2.57 & 1.62 \\
Walking ability & 1.48 & 1.61 \\
Normal work & 1.68 & 1.55 \\
Relations with others & 0.90 & 1.30 \\
Sleep & 1.62 & 1.63 \\
Enjoyment of life & 1.47 & 1.59 \\
Overall interference & Median (IQR): 3(I.5-4.5) & Range: 0-9 \\
\hline
\end{tabular}

Abbreviation: IQR, interquartile range.

While high pain prevalence among the elderly and an increased burden related to pain among people in low socioeconomic status have been widely studied, this study expands evidence of the high prevalence of pain among a young adult population attending university. The prevalence of pain was $73.5 \%$ among university students in this study. This is higher compared to a previous study on university students in South Korea reporting $46.3 \%$ of pain prevalence. ${ }^{27}$ This discrepancy may be due to the previous study specifically investigating joint or muscle pain, whereas the current study included pain complaints arising from any body area. The current study's results are comparable to the previously reported pain prevalence of $74.4 \%$ investigating musculoskeletal pain among university students in Pakistan. ${ }^{19}$ An additional study reported pain prevalence of $92.7 \%$ in university students in Hong Kong. ${ }^{24}$ In Norway, the prevalence of chronic pain lasting more than 3 months among university students was $54 \%,{ }^{20}$ whereas it was $7.8 \%$ in the current study. It is difficult to directly compare these results among studies since the specific criteria used to measure prevalence of pain are not consistent across the studies. Reported pain prevalence in previous studies varied greatly $(40-90 \%)$ by participant characteristics and study locations. Preliminarily, these results may suggest that pain perception or self-report differs across ethnic and cultural groups even in young adults. Indeed, pain is influenced by many factors, and race/ethnicity and culture are among those infrequently investigated. ${ }^{31}$ Beliefs about pain developed within an environment of ethnicity and culture may influence pain management and treatment, and this may influence differences in the prevalence of pain across ethnic groups. ${ }^{32}$

The most frequently reported painful body areas in this study among the university students were the shoulders and neck, consistent with previous reports. ${ }^{24}$ This may be associated with mobile phone or extended computer use as technology use is common and frequent in university students. In fact, previous studies reported a significant positive correlation between the duration of mobile phone use and severity and duration of neck pain. ${ }^{21,28,29}$ Headache was also common (45.8\%), and students indicated that one of the most associated factors with pain was stress in the current study (data not shown). Furthermore, the more advanced in school the student was, the greater the prevalence of self-reported pain in this study. These

Table 5 Factors Associated with Self-Reported Negative Impact of Pain Among University Students Who Reported Experiencing Pain During Last 6 Months $(\mathrm{N}=297)$

\begin{tabular}{|l|c|c|c|c|}
\hline Characteristics & Adjusted OR & Standard Error & $\mathbf{P}$ & $\mathbf{9 5 \%} \mathbf{C l}$ \\
\hline Gender (female) & 1.17 & 0.504 & 0.713 & $0.504,2.721$ \\
\hline Major (health related) & 1.49 & 0.559 & 0.281 & $0.719,3.109$ \\
\hline Painful body areas (>5) & 2.98 & 1.106 & 0.003 & $1.442,6.168$ \\
\hline $\begin{array}{l}\text { Pain duration* } \\
\text { I-7 days }\end{array}$ & 3.01 & 1.328 & 0.013 & $1.265,7.146$ \\
More than a week & 4.46 & 2.395 & 0.005 & $1.555,12.774$ \\
\hline Pain intensity & 1.67 & 0.184 & $<0.001$ & $1.346,2.073$ \\
\hline Use of OTC medication & 1.54 & 0.576 & 0.246 & $0.741,3.207$ \\
\hline
\end{tabular}

Note: *Reference group: less than one day.

Abbreviations: OR, odds ratio; $\mathrm{Cl}$, confidence interval; OTC, over the count. 
results suggest that the high prevalence of pain among university students may be associated with their lifestyles and habits, along with stress related to academic achievement.

Previous reports indicate the cultural tendency for Asian populations to believe pain is a sign of weakness, leading to a reluctance to report pain associated with stigmatization. ${ }^{5,33}$ Consistent with this hypothesis, a crosscultural comparison study of experimental pain indicated that Asians had greater pain tolerance compared to nonHispanic Whites. ${ }^{2}$ Additional explanations of higher pain tolerance in Asians may be explained by Asians' predisposition to demonstrate inner strength by accepting pain and suffering for spiritual growth. ${ }^{32}$ However, as evidenced in the current study, these predispositions may not be true for young adults. The average pain intensity in this study was $4.8 / 10$ and is similar to previous studies from the Middle East. ${ }^{21}$ Furthermore, a recent study of Asian students from Hong Kong reported an average pain intensity of 3.4/10. ${ }^{24}$ This indicates that healthcare professionals should be aware of differences in the tendency to report pain across different ethnic groups, as well as intraracial differences by age group. This may be especially relevant when younger caregivers and health care professionals are caring for older adults.

The university students in this study reported nonpharmacological pain management methods as the most frequently used method for pain control. Among subtypes of non-pharmacological methods, rest and massage were used most commonly and were perceived as the most effective among non-pharmacological methods used. Interestingly, the use of alternative medicine, including acupressure, acupuncture, or TCM, was not as frequently reported by South Korean university students. This is in contrast to previous research on the prevalence of these pain management methods among Asians when compared to other pain management methods, including OTC analgesics. ${ }^{5}$ In fact, more than half of the participants who experienced pain made use of OTC medications to manage their pain in this study. These findings suggest that the influence of culture on pain management differs by age group. Further studies exploring factors influencing pain management choice specifically comparing culture and age are warranted.

Pain experiences among university students have been associated with poor mental health outcomes, in the context of a population particularly vulnerable to mental health problems. ${ }^{34} \mathrm{~A}$ recent study indicated that worry and rumination induced by pain were associated with negative mood, including depression and anxiety among university students. ${ }^{7}$ Consistent with these findings, pain interference in this study was greatest for mood. Factors associated with greater pain interference in this study were longer duration of pain experience, more widespread bodily pain, and greater pain intensity.

While the current study revealed some novel findings and supported some previous research results concerning pain among university students, this study has several limitations. First, participants were recruited using a convenience sampling method; therefore, generalization of the findings may be limited. Second, the measures used for this study have not been previously used in the Korean population. However, these measures showed good reliability and validity when they were validated in Asians, ${ }^{24}$ and results showed good internal consistency in the present study. Further studies comparing pain and associated factors across diverse ethnic groups are warranted using more extensive multi-dimensional pain measures. Future studies should include other factors (eg, psychological and socioeconomic status) known to influence pain and the presence of comorbid chronic conditions as well as social support factors that influence pain coping and interference. Further studies examining the effects of age and culture on the efficacy of specific pharmacological and nonpharmacological pain management methods are necessary to build evidence for individualized treatment regimes. Pain management is influenced by many factors, including origin of pain (musculoskeletal, neuropathic or visceral), pain duration, and patient understanding of pharmacologi$\mathrm{cal} /$ non-pharmacological strategies. Studies investigating pain management strategies in university students are suggested for future studies to optimize pain management.

\section{Clinical Implications}

Based on the findings of high prevalence of pain among university students and its evident and potential negative impact in this particular age group, we suggest clinicians who care for young adult patients should include pain assessment in their practice. Providing education programs to enhance knowledge about pain and its impact on mental health is necessary for this group. Appropriate pharmacological pain management programs with assessment of pain medication-related knowledge for this young adult population are necessary as they prefer using medications for pain control to avoid opioid misuse and OTCmedication overuse. Rest and massage were found to be 
favorable non-pharmacological methods of pain control in this study. Providing student common rooms with safe equipment (eg, massage chairs) and providing counseling services for mood disturbance in university students may be beneficial for pain management, ultimately leading to better mental and physical health.

\section{Conclusion}

Given the high prevalence of pain in university students, early detection by pain assessment as well as appropriate educational programs targeting pain management is necessary to reduce the potential negative impact of pain. University students reporting multiple painful body areas with long-term and high-intensity pain should receive effective care to prevent long-term negative effects of high pain interference, including disability and poor mental health. When developing pain management programs, cross-cultural as well as intra-ethnic differences should be considered.

\section{Disclosure}

The authors declare no conflicts of interest.

\section{References}

1. Raja SN, Carr DB, Cohen M, et al. The revised international association for the study of pain definition of pain: concepts, challenges, and compromises. Pain. 2020;161(9):1976-1982. doi:10.1097/i. pain.0000000000001939

2. Kim HJ, Yang GS, Greenspan JD, et al. Racial and ethnic differences in experimental pain sensitivity: systematic review and meta-analysis. Pain. 2017;158(2):194-211. doi:10.1097/j.pain.0000000000000731

3. GBD Causes of Death Collaborators. Global, regional, and national age-sex-specific mortality for 282 causes of death in 195 countries and territories, 1980-2017: a systematic analysis for the global burden of disease study 2017. Lancet. 2018;392(10159):1789-1858. doi:10.1016/0140-6736(18)32203-7

4. Bostrom M, Dellve L, Thomee S, Hagberg M. Risk factors for generally reduced productivity - a prospective cohort study of young adults with neck or upper-extremity musculoskeletal symptoms. Scand J Work Environ Health. 2008;34(2):120-132. doi:10.5271/ sjweh.1218

5. Carteret M. Cultural aspects of pain management. Available from: https://www.dimensionsofculture.com/2010/11/cultural-aspects-ofpain-management/. Accessed January 8, 2021.

6. Jahre H, Grotle M, Smedbråten K, Dunn KM, Øiestad BE. Risk factors for non-specific neck pain in young adults. A systematic review. $B M C$ Musculoskelet Disord. 2020;21(366):1-12. doi:10.1186/s12891-02003379-y

7. Rogers AH, Bakhshaie J, Ditre JW, et al. Worry and rumination: explanatory roles in the relation between pain and anxiety and depressive symptoms among college students with pain. $\mathrm{J} \mathrm{Am} \mathrm{Coll} \mathrm{Health.}$ 2019;67(3):275-282. doi:10.1080/07448481.2018.1481071

8. Potur DC, Bilgin NC, Komurcu N. Prevalence of dysmenorrhea in university students in Turkey: effect on daily activities and evaluation of different pain management methods. Pain Manag Nurs. 2014;15 (4):768-777. doi:10.1016/j.pmn.2013.07.012
9. O'Connell K, Davis AR, Westhoff C. Self-treatment patterns among adolescent girls with dysmenorrhea. J Pediatr Adolesc Gynecol. 2006;19(4):285-289. doi:10.1016/j.jpag.2006.05.004

10. Mitka M. When teens self-treat headaches, OTC drug misuse is frequent result. JAMA. 2004;292(4):424-425. doi:10.1001/ jama.292.4.424

11. Pereira FS, Bucaretchi F, Stephan C, Cordeiro R. Self-medication in children and adolescents. J Pediatr (Rio J). 2007;83(5):453-458. doi:10.2223/JPED. 1703

12. Cleeland CS. The brief pain inventory, user guide. Available from: https://www.mdanderson.org/documents/Departments-and-Divisions /Symptom-Research/BPI_UserGuide.pdf. Accessed January 14, 2021.

13. Uçar T, Timur Taşhan S, Aksoy Derya Y, Nacar G. An analysis of dysmenorrhoea and depressive symptoms in university students: a case-control study. Int $J$ Nurs Pract. 2018;24(5):e12678. doi:10.1111/ijin. 12678

14. Hu Z, Tang L, Chen L, Kaminga AC, Xu H. Prevalence and risk factors associated with primary dysmenorrhea among Chinese female university students: a cross-sectional study. $J$ Pediatr Adolesc Gynecol. 2020;33(1):15-22. doi:10.1016/j.jpag.2019.09.004

15. Fernández-Martínez E, Onieva-Zafra MD, Parra-Fernández ML. Lifestyle and prevalence of dysmenorrhea among Spanish female university students. PLoS One. 2018;13(8):e0201894. doi:10.1371/ journal.pone.0201894

16. Orhan C, Çelenay ŞT, Demirtürk F, Özgül S, Üzelpasacı E, Akbayrak T. Effects of menstrual pain on the academic performance and participation in sports and social activities in Turkish university students with primary dysmenorrhea: a case control study. $J$ Obstet Gynaecol. 2018;44(11):2101-2109. doi:10.1111/jog.13768

17. Abu Helwa HA, Mitaeb AA, Al-Hamshri S, Sweileh WM. Prevalence of dysmenorrhea and predictors of its pain intensity among Palestinian female university students. BMC Womens Health. 2018;18(1):1-11. doi:10.1186/s12905-018-0516-1

18. Smiljic S, Savic S, Stevanovic J, Kostic M. Prevalence and characteristics of orofacial pain in university students. J Oral Sci. 2016;58 (1):7-13. doi:10.2334/josnusd.58.7

19. Haroon H, Mehmood S, Imtiaz F, Ali SA, Sarfraz M. Musculoskeletal pain and its associated risk factors among medical students of a public sector University in Karachi, Pakistan. J Pak Med Assoc. 2018;68(4):682-688.

20. Grasdalsmoen M, Engdahl B, Fjeld MK, et al. Physical exercise and chronic pain in university students. PLoS One. 2020;15(6):e235419. doi:10.1371/journal.pone.0235419

21. Al-Hadidi F, Bsisum I, AlRyalat SA, et al. Association between mobile phone use and neck pain in university students: a cross-sectional study using numeric rating scale for evaluation of neck pain. PLoS One. 2019;14(5):e0217231. doi:10.1371/journal. pone. 0217231

22. Osama M, Ali S, Malik RJ. Posture related musculoskeletal discomfort and its association with computer use among university students. J Pak Med Assoc. 2018;68(4):639-641.

23. Kędra A, Kolwicz-Gańko A, Sitarski D, Ewertowska P, Czaprowski D. Low back pain and everyday functioning of students. Ortop Traumatol Rehabil. 2016;18(1):31-39. doi:10.5604/ 15093492.1198840

24. Tse MMY, Tang A, Budnick A, Ng SSM, Yeung SSY. Pain and pain management among university students: online survey and web-based education. Cyberpsychol Behav Soc Netw. 2017;20 (5):305-313. doi:10.1089/cyber.2016.0580

25. Morais BX, Dalmolin GL, Andolhe R, Dullius AIS, Rocha LP. Musculoskeletal pain in undergraduate health students: prevalence and associated factors. Rev ESC Enferm USP. 2019;15:e03444. doi:10.1590/S1980-22X2018011403444

26. Wristen BG, Fountain SE. Relationships between depression, anxiety, and pain in a group of university music students. Med Probl Perform Art. 2013;28(3):152-158. doi:10.21091/mppa.2013.3030 
27. Ahn S-H, Um Y-J, Kim Y-J, et al. Association between physical activity levels and physical symptoms or illness among university students in Korea. Korean J Fam Med. 2016;37(5):279-286. doi:10.4082/kjfm.2016.37.5.279

28. Chu MK, Song HG, Kim C, Lee BC. Clinical features of headache associated with mobile phone use: a cross-sectional study in university students. BMC Neurol. 2011;11(1):1-7. doi:10.1186/1471-237711-115

29. Kim H-J, Kim J-S. The relationship between smartphone use and subjective musculoskeletal symptoms and university students. J Phys Ther Sci. 2015;27(3):575-579. doi:10.1589/jpts.27.575

30. Dol KS. Fatigue and pain related to Internet usage among university students. J Phys Ther Sci. 2016;28(4):1233-1237. doi:10.1589/ jpts. 28.1233
31. Miller ET, Abu-Alhaija DM. Cultural influences on pain perception and management. Pain Manag Nurs. 2019;20(3):183-184. doi:10.1016/j.pmn.2019.04.006

32. Kawi J, Reyes AT, Arenas RA. Exploring pain management among Asian immigrants with chronic pain: self-management and resilience. J Immigr Minor Health. 2019;21(5):1123-1136. doi:10.1007/s10903018-0820-8

33. Dhatt G, Simpson P, MacDonald V. Self-management of osteoarthritis: a culturally-specific chronic care model for South Asians. Int J Orthop Trauma Nurs. 2012;16(3):21-127. doi:10.1016/j.ijotn.2012.01.001

34. Borgman S, Ericsson I, Clausson EK, Garmy P. The relationship between reported pain and depressive symptoms among adolescents. $J$ Sch Nurs. 2020;36(2):87-93. doi:10.1177/ 105984051878007

\section{Publish your work in this journal}

The Journal of Pain Research is an international, peer reviewed, open access, online journal that welcomes laboratory and clinical findings in the fields of pain research and the prevention and management of pain. Original research, reviews, symposium reports, hypothesis formation and commentaries are all considered for publication. The manuscript management system is completely online and includes a very quick and fair peer-review system, which is all easy to use. Visit http:// www.dovepress.com/testimonials.php to read real quotes from published authors. 